Artículo científico

(Original paper)

\title{
DIVERSIDAD DE AVES EN SITIOS CON DISTINTO USO DE SUELO EN NUEVO CONHUAS, CALAKMUL, MÉXICO
}

\author{
BIRD DIVERSITY IN SITES WITH DIFFERENT LAND USE IN NUEVO CONHUAS, \\ CALAKMUL, MEXICO
}

\section{FILIBERTo GONZÁLEZ-MARTÍN DEL CAMPO ${ }^{1 *}$, DARIo ALEJANDRo NAVARRETE-GUTIÉRREZ ${ }^{1,2}$, PAULA L. ENRÍQUEZ ${ }^{1}$, MARÍA GUADALUPE GORDILLO-PÉREZ ${ }^{3}$}

\begin{abstract}
${ }^{1}$ Departamento de Conservación de la Biodiversidad, El Colegio de La Frontera Sur, Periférico Sur s/n, María Auxiliadora, 29290 San Cristóbal de las Casas, Chiapas. <fili.gmd@gmail.com>; <penrique@ecosur.mx>

${ }^{2}$ El Colegio de La Frontera Sur, Laboratorio de Análisis de Información Geográfica y Estadística, Periférico Sur s/n, María Auxiliadora, San Cristóbal de las Casas, Chiapas, 29290, México. <dnavarre@ecosur.mx>

${ }^{3}$ IMSS/Centro Médico Nacional Siglo XXI, Hospital de Pediatría, Unidad de investigación médica de enfermedades infecciosas y parasitarias, Avenida Cuauhtémoc 330, Doctores, 06720 Cuauhtémoc, CDMX. 〈lugope2@yahoo.com.mx>

*Autor de correspondencia: <fili.gmd@gmail.com>
\end{abstract}

Recibido: 16/06/2018; aceptado: 28/10/2019; publicado en línea: 15/11/2019

Editor responsable: Ricardo Rodríguez Estrella

González-Martín del Campo, F., Navarrete-Gutiérrez, D. A., Enríquez, P. L., Gordillo-Pérez, G. (2019) Diversidad de aves en sitios con distinto uso de suelo en Nuevo Conhuas, Calakmul, México. Acta Zoológica Mexicana (nueva serie), 35, 1-18. https://doi.org/10.21829/azm.2019.3501233

RESUMEN. Las aves son uno de los grupos de vertebrados más importantes en el funcionamiento de los ecosistemas al cumplir con importantes funciones como la polinización o dispersión de semillas. Sin embargo, las comunidades y sus poblaciones se pueden ver afectadas por cambios en los ecosistemas. Estas modificaciones pueden ser reflejo de los diferentes usos de la tierra, por lo que es importante analizar cómo las áreas con diferentes usos de suelo y coberturas influyen en las comunidades de aves. Para este estudio, realizamos muestreos de aves de febrero a junio de 2017 utilizando redes de niebla en cuatro sitios con distintas condiciones de uso: un sitio con vegetación conservada (Reserva de la Biósfera de Calakmul), dos acahuales, uno agrícola, otro forestal y un sitio ganadero. La diversidad de las comunidades fue diferente en cada uno de los sitios muestreados, se identificó que las comunidades de aves en los acahuales y la Reserva de la Biósfera de Calakmul son más similares a las del sitio ganadero. El gremio de los semilleros y las aves generalistas fueron más abundantes en el sitio ganadero. El 39\% de las especies estuvo presente en la reserva de Calakmul, que fue el sitio con la comunidad más diversa $\left(D_{1}=36.38\right)$; el sitio ganadero estuvo dominado por un menor número de especies $\left(\mathrm{D}_{2}=3.88\right)$. Los análisis multivariados mostraron que el tipo de vegetación presente en los sitios influye en la abundancia de familias y especies de aves (Dev= 373.1, $p=0.001$ ). La presencia de especies compartidas entre los acahuales y la Reserva de la Biósfera de Calakmul demuestra la importancia en la conservación y regeneración de acahuales ya que son estados de transición que ayudan a recuperar especies presentes en las selvas maduras y que se encuentren bajo alguna categoría de riesgo.

Palabras clave: Diversidad; comunidades; avifauna; uso de suelo; Calakmul 
González-Martín del Campo, F., Navarrete-Gutiérrez, D. A., Enríquez, P. L., Gordillo-Pérez, G. (2019) Bird diversity in sites with different land use in Nuevo Conhuas, Calakmul, Mexico. Acta Zoológica Mexicana (nueva serie), 35, 1-18. https://doi.org/10.21829/azm.2019.3501233

\begin{abstract}
Birds are one of the most important vertebrate groups for the ecosystems' functioning, because their functions include pollination and seed dispersal. Nevertheless, their communities and populations are affected by changes in the ecosystems. These modifications can be a reflection of land use, and for this reason it is important to analyze how sites with different land use and ground cover can have an influence on the birds' communities. For this study, we sampled birds from February to June 2017 using mist nets in sites with different land use conditions: a site inside the Calakmul Biosphere Reserve, two acahuales, one agricultural and one forested, and one livestock site. The diversity of communities was different in each one of the sampling sites. We identified that bird communities between acahuales and the Calakmul Biosphere Reserve had a greater similarity than the livestock site. The seeders guild and the generalist species were more abundant in the livestock site. There was a greater number of specialist birds with low abundances in the Calakmul Biosphere Reserve. 39\% of the species were present in the Calakmul Biosphere Reserve, that was the site with the greater diversity $\left(D_{1}=36.38\right)$, and livestock site was dominated by a lower number of species $\left(\mathrm{D}_{2}=3.88\right)$. The multivariate analysis showed that the vegetation type present in the sites influence the abundance of the bird species ( $\mathrm{Dev}=373.1, p=0.001)$, and their families. The presence of shared species between the acahuales and the Calakmul Biosphere Reserve shows the importance of the conservation and regeneration of the acahuales because they are a transition stage that contributes to the recovery of some bird species present in mature jungles that are threatened or belong to a risk category.
\end{abstract}

Key words: Diversity; communities; avifauna; land use, Calakmul

\title{
INTRODUCCIÓN
}

México presenta una elevada diversidad de aves, ya que aproximadamente se distribuyen 1,115 especies que representan el 10\% de la diversidad mundial (Berlanga et al., 2017). La península de Yucatán y la vertiente del Pacífico son las áreas dónde se concentra una mayor riqueza (Navarro et al., 2014). La mayoría de las aves son de hábitos terrestres y cumplen con distintas funciones ecológicas como la dispersión de semillas y polinización, por esta razón son consideradas como "ingenieros de los ecosistemas" (Sekercioglu, 2006); son los vertebrados terrestres con la mayor capacidad de movimiento y que pueden cubrir amplias distancias durante diferentes etapas de su historia de vida (Cox, 2010). Para muchas especies la migración es parte de su historia de vida y es considerado un proceso ecológico-evolutivo (Newton, 2008), en el que las aves tienen una respuesta adaptativa a la heterogeneidad espacial y temporal en los recursos.

México ha sufrido una de las mayores tasas de modificación de sus ecosistemas y deforestación a nivel de América Latina, tan solo en las últimas dos décadas más de 100,000 ha de bosques han sido deforestados en México (Ellis et al., 2017). Entre 1993 y 2002 Campeche tenía una tasa de deforestación de 30,968 ha por año (Céspedes \& Moreno, 2010), causada por la intensificación de actividades agropecuarias (Smith et al., 2001). En Campeche el 74\% del territorio estatal corresponde a terrenos que han sido modificados por actividades humanas y, solamente el $25.1 \%$ restante está cubierto por vegetación natural (INEGI, 2015). En la región de Calakmul, Campeche, el sistema de propiedad ejidal ha sido el principal promotor del cambio de uso de suelo en la región (Chowdhury, 2006). El resultado es un paisaje manejado conteniendo un mosaico de distintos tipos de vegetación con diferentes etapas de sucesión (Warkentin et al., 1995; Smith et al., 2001). Un ejemplo de esto es la presencia de acahuales o hubchés (Martínez \& Galindo, 2002), que son comunidades secundarias de la selva baja o mediana asociadas a la producción agrícola de la roza-tumba y quema (Levy \& Aguirre, 2000). Algunas comunidades de la región reconocen al menos cuatro estadios dependiendo de su edad, en los cuáles la estructura y tipo de vegetación varía 
dependiendo del tiempo de abandono (Granados et al., 1999). Cuando estas prácticas se realizan a pequeña escala y quedan parches intactos de vegetación se puede promover el mantenimiento de la biodiversidad (Smith et al., 2001). En este sentido, se ha considerado que los disturbios intermedios producen un mosaico de hábitats que, en paisajes transformados, pueden tener una diversidad de especies mayor. Esta hipótesis considera que en pequeños disturbios la exclusión competitiva produce una menor diversidad, mientras que, en disturbios grandes, las especies suelen ser extirpadas por no poder colonizar nuevos espacios. Otra condición que se menciona facilita este incremento en la diversidad es la heterogeneidad en el paisaje, siendo de alguna manera importante para la supervivencia de distintas comunidades animales (Fahrig, 2003, 2017; Connell, 1978) entre ellas, las aves. Sin embargo, esta hipótesis ha sido debatida ya que son pocos los estudios que han demostrado que los valores más altos de diversidad se presenten en niveles intermedios de disturbio (Fox, 2013). La vegetación presente en los sitios como resultado del uso de suelo, puede relacionarse con la presencia de distintos grupos de aves, como es el caso del uso de los cafetales por aves migratorias, o los paisajes agroforestales en los que las aves utilizan preferentemente el dosel (Greenberg et al., 1997; Van et al., 2008).

Es importante identificar la influencia del uso del suelo sobre las comunidades animales, ya que puede ayudar a identificar zonas necesarias para la conservación de especies residentes y migratorias. El objetivo general de este estudio fue analizar la influencia del uso de suelo sobre las comunidades de aves en el ejido Nuevo Conhuas, en la región de Calakmul. Los objetivos particulares fueron: determinar la cobertura de suelo de cada sitio, como reflejo de su uso; analizar la diversidad de la comunidad de aves en cada sitio con distinto uso de suelo; analizar la influencia del uso de suelo sobre la abundancia de especies entre sitios y mes de muestreo, así como la abundancia de las aves migratorias neotropicales y residentes; analizar la influencia del uso de suelo sobre gremios alimenticios; identificar aquellas especies que se obtuvieron en una sola ocasión o aquellas obtenidas en dos ocasiones a lo largo del muestreo; e identificar la importancia de cada uso de suelo sobre distintas especies y familias de aves.

\section{MATERIALES Y MÉTODOS}

El ejido Nuevo Conhuas se localiza al sureste del estado de Campeche, (89 55' 22" N, $\left.18^{\circ} 32^{\prime} 26^{\prime \prime} \mathrm{O}\right)$ en el municipio de Calakmul, y forma parte de la Reserva de la Biósfera de Calakmul, la cual limita al este con el estado de Quintana Roo y al sur con la República de Guatemala (Fig. 1). De acuerdo con el sistema de clasificación de Köppen modificado por García (1973), el clima de la región es cálido subhúmedo (Aw) (García, 1973; SEMARNAP, 1999), la temperatura promedio anual es de $24.6^{\circ} \mathrm{C}$, y las precipitaciones pueden alcanzar hasta $873.3 \mathrm{~mm}$ (SEMARNAP, 1999). La vegetación principal son selvas secas inmersas en una mezcla de selvas tropicales, subperennifolias y subcaducifolias secas pudiendo ser altas, medianas, bajas o inundables (Miranda, 1958; SEMARNAP, 1999).

Selección de sitios de muestreo. El muestreo fue realizado en el ejido Nuevo Conhuas $\left(18^{\circ} 32^{\prime} 26^{\prime \prime} \mathrm{N}, 89^{\circ}\right.$ $55^{\prime} 22$ " O) donde se seleccionaron cuatro sitios con base en el uso actual de suelo. Para esto, realizamos entrevistas semiestructuradas a tres de los pobladores más viejos del ejido, quienes fueron de los primeros habitantes del mismo y conocieron las condiciones originales de la región de estudio y los cambios que ha habido en el uso del suelo. El primer sitio se localizó dentro de la REBICA (18 $18^{\prime} 52.19^{\prime \prime}$ N, 89 51' 23.40" $\mathrm{O})$, esta área está sujeta a protección y no se permite el aprovechamiento extractivo desde la declaración de la reserva en 1989.

La vegetación presente en este sitio es una mezcla de selva baja (también llamados bajos inundables), selva mediana y alta. Los otros sitios se ubicaron en la zona de amortiguamiento de la REBICA; en estos sitios se lleva a cabo aprovechamiento de recursos forestales y actividades como la agricultura y ganadería. El segundo sitio es un acahual de manejo forestal con más de 20 años de abandono (18 $31^{\prime}$ $19.61 " \mathrm{~N}, 89^{\circ} 47^{\prime} 31.92^{\prime \prime}$ O) en el que se realizó la explotación de los recursos forestales, la vegetación es 
selva mediana. El tercer sitio es un acahual de agricultura con vegetación secundaria entre 5 y 10 años desde su abandono ( $18^{\circ} 32^{\prime} 9.24^{\prime \prime} \mathrm{N}, 89^{\circ} 53^{\prime} 12.95^{\prime \prime} \mathrm{O}$ ), era sembrado con maíz, chile y calabaza (chihua). El tipo de agricultura que se realizó en este sitio fue roza, tumba y quema; la vegetación presente actualmente es selva baja y selva mediana. El cuarto sitio es de manejo ganadero ( $18^{\circ} 31^{\prime} 22.97^{\prime \prime} \mathrm{N}, 89^{\circ} 54^{\prime} 47.53$ " O) en el que se mantiene ganado bovino en pastizales de Muhlenbergia spp. Es importante mencionar que los sitios en la zona de amortiguamiento se encuentran inmersos en parches en los que se han realizado distintas actividades de aprovechamiento, tales como ganadería, agricultura, aprovechamiento forestal y apicultura, entre otras. Algunas actividades se siguen realizando, como la agricultura a pequeña escala y apicultura.

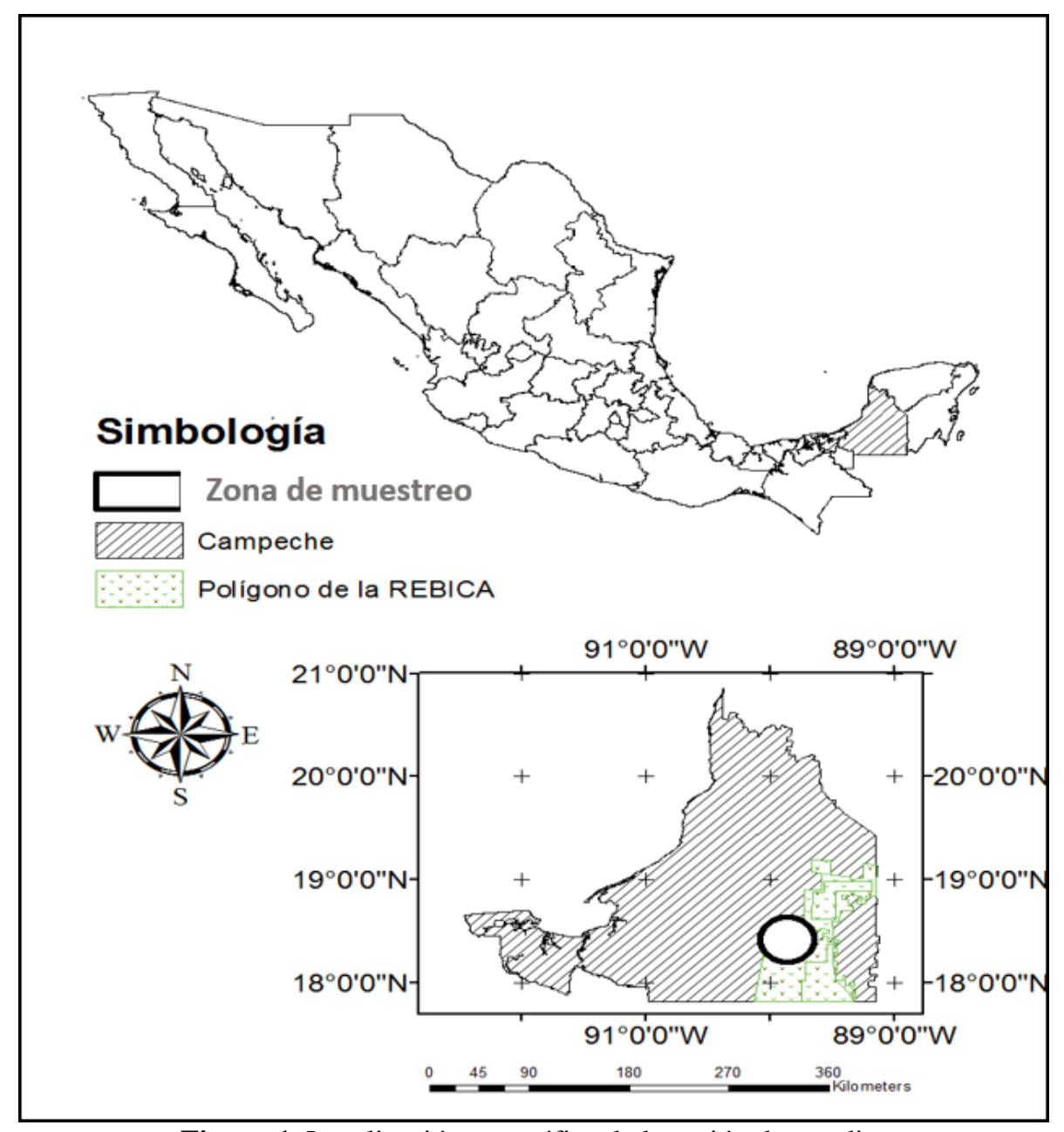

Figura 1. Localización geográfica de la región de estudio.

Muestreo de aves. El muestreo de aves se realizó del 01 de febrero de 2017 al 08 de junio de 2017. Se colocaron seis redes de niebla en el sotobosque, tres de seis $\mathrm{m}$ y tres de $12 \mathrm{~m}$ con una separación de 90 y $120 \mathrm{~m}$ entre ellas (Ralph et al., 2001), las cuales permanecieron abiertas desde las 06:30 hr hasta las 11:00 hr durante tres días consecutivos en las fechas anteriormente mencionadas. Este procedimiento se realizó cinco veces en cada sitio con un intervalo de tiempo de tres semanas. El esfuerzo total de muestreo fue de $14,580 \mathrm{hr} / \mathrm{red}$, las aves fueron identificadas a nivel de especie con guías de campo (Kaufman, 2005; Perlo, 2006; MacKinnon, 2013; Arizmendi \& Berlanga, 2014; Sibley, 2014). Las aves fueron marcadas en ambos tarsos con barniz para uñas (Kendall et al., 2009) para identificar posibles recapturas. Posterior a esto las aves fueron liberadas en el lugar de captura. 
Análisis del paisaje. Para determinar la cobertura del suelo de cada sitio se utilizó ArcGIS versión 10.2. y FRAGSTATS versión 4 (McGarigal et al., 2012). Fue utilizada una imagen satelital LANDSAT 8 OLI del 14 de febrero de 2017 (USGS, 2017); debido a que no se requería hacer la comparación en el tiempo de las coberturas y usos de suelo de la zona, no se realizó una corrección atmosférica y la imagen se trabajó utilizando los números digitales. Se llevó a cabo una clasificación automatizada no supervisada determinando cuatro clases para toda la imagen (con base en el conocimiento de la región de estudio por el primer autor). Las clases fueron clasificadas de la siguiente manera: vegetación conservada (VEC), acahuales maduros (ACM), vegetación secundaria y pastizales (VESPA), y caminos y construcciones (SINV). Fue calculada también la riqueza y número de parches, así como la densidad de borde de los sitios de estudio con FRAGSTATS v4 (McGarigal et al., 2012). Se incluyó también la distancia a la comunidad en kilómetros (DISC) como variable explicativa.

Se estableció una zona buffer de 70 ha a partir del centroide de cada sitio en la que fueron colocadas las redes, la cual fue determinada con base en datos de rango hogareño de tres especies capturadas: Hylocichla mustelina (Gmelin, 1789), Sittasomus griseicapillus (Vieillot, 1818) y Empidonax virescens (Vieillot, 1819). Se ha reportado que los ámbitos hogareños registrados para estas especies no exceden una superficie de 70 ha (Anders et al., 1998; Ausprey \& Rodewald, 2013; Jirinec et al., 2016).

Análisis de datos. La diversidad de aves fue analizada con el programa estadístico R Studio 1.0.153 (RStudio Team, 2016) utilizando el Paquete INEXT para conocer los números efectivos de especies (Chao et al., 2014) en sus órdenes (q) 0,1 y 2, con el fin de identificar las diferencias en la diversidad de especies en cada condición de uso de suelo. Los números efectivos de especies permiten comparar la magnitud de las diferencias en las comunidades (Moreno et al., 2010). Fue calculado el valor de equidad $\mathrm{EF}_{1,2}=\mathrm{D}_{2} / \mathrm{D}_{1}$ ( $\mathrm{EF}=$ equidad; $\mathrm{D}_{2}=$ orden dos de diversidad; $\mathrm{D}_{1}=$ Orden uno de diversidad) con los números efectivos de especies; esta relación permite representar la proporción de especies dominantes de una comunidad (Jost, 2010). Se calculó la cobertura de muestra para la comunidad de aves, para realizar una comparación más acertada en relación con los valores de diversidad obtenidos por medio de los números efectivos de especies (Jost, 2010).

Fue calculado el índice de diversidad Beta de Whittaker $(\beta \mathrm{w})$, el cual es una relación del promedio de las diversidades alfa y la diversidad gamma (Calderón et al., 2012), así como el índice de similitud de Jaccard para conocer la similitud de las comunidades en cada sitio de muestreo. Fueron utilizadas pruebas de Kruskal-Wallis con la corrección de Bonferroni en R Studio 1.0.153 para analizar las diferencias en la abundancia entre los sitios muestreados y meses de muestreo para aves migratorias neotropicales y aves residentes, así como entre los tres gremios alimenticios más abundantes de la comunidad (insectívoros, nectarívoros y semilleros). La determinación de los gremios fue realizada con información disponible en los sitios web Naturalista (htp://www.naturalista.mx), Guía en línea del Laboratorio de aves de Cornell (http://neotropical.birds.cornell.edu) y guía en línea de Audubon para aves de Norteamérica (http://www.audubon.org/bird-guide), así como información en Neri (2007) y la guía de aves de Sibley (2014). Durante el muestreo basado en la abundancia de las especies, y con el objetivo de tener una aproximación sobre la presencia de especies raras, identificamos aquellas especies únicas o que se registraron en solo una unidad de muestreo ( $\mathrm{f} 1$ o singletons) y aquellas especies que se capturaron en dos unidades muestrales durante todo el estudio (f2 o doubletons), con el programa estadístico EstimateS V.9 (Colwell, 2013).

Se realizó un análisis de correspondencia canónica (CCA) con el paquete Vegan (Oksanen et al., 2018) en R Studio, para determinar las asociaciones entre la comunidad de aves y las variables de tipos de vegetación de los sitios muestreados. Este método multivariado evalúa las relaciones de las especies con el ambiente, que están interactuando en un área determinada (Braak, 1987). Las variables utilizadas en el modelo fueron el logaritmo base 10 de la superficie de acahuales maduros (ACM), caminos y construcciones (SINV), y la distancia a la comunidad de Nuevo Conhuas (DISC). La abundancia de especies fue 
transformada al logaritmo natural más uno, la significancia del modelo y de las variables incluidas fueron evaluadas con pruebas ANOVA. Para la ordenación se consideró la escala de Hill tipo 2, (el promedio ponderado de las especies por sitio de registro). Esta escala permite interpretar espacialmente la relación entre las especies y las variables ambientales en los sitios donde éstas se registraron (McCune \& Grace, 2002). Debido a que el CCA es sensible a la abundancia de las especies menos abundantes o raras, se realizó el tratamiento previo de los datos de abundancias de las especies de aves, transformando la variable considerando la distancia de Hellinger por ser recomendada para análisis de conglomerados o de ordenación cuando se analiza la composición de comunidades (Legendre \& Gallagher, 2001).

Con las variables significativas del CCA se realizaron modelos lineales generalizados (GLM) con distribución binomial negativa para la comunidad de aves, con el objetivo de analizar la influencia del sitio y tipos de vegetación sobre la abundancia de las aves y familias; las familias representadas por un solo individuo fueron excluidas del modelo. Todos los modelos lineales generalizados se realizaron en $\mathrm{R}$ studio 1.0.153 con el paquete mvabund (Wang et al., 2012).

\section{RESULTADOS}

La REBICA presentó la mayor superficie de vegetación conservada (52.56 ha) y la menor la tuvo el sitio ganadero (3.96 ha). Los sitios identificados como acahuales presentaron superficies similares de todos los tipos de vegetación. Fueron la REBICA y el sitio ganadero los que presentaron superficies de vegetación más contrastantes. El sitio dentro de la REBICA fue el más alejado de la comunidad, mientras que el sitio ganadero fue el más cercano (Fig. 2).

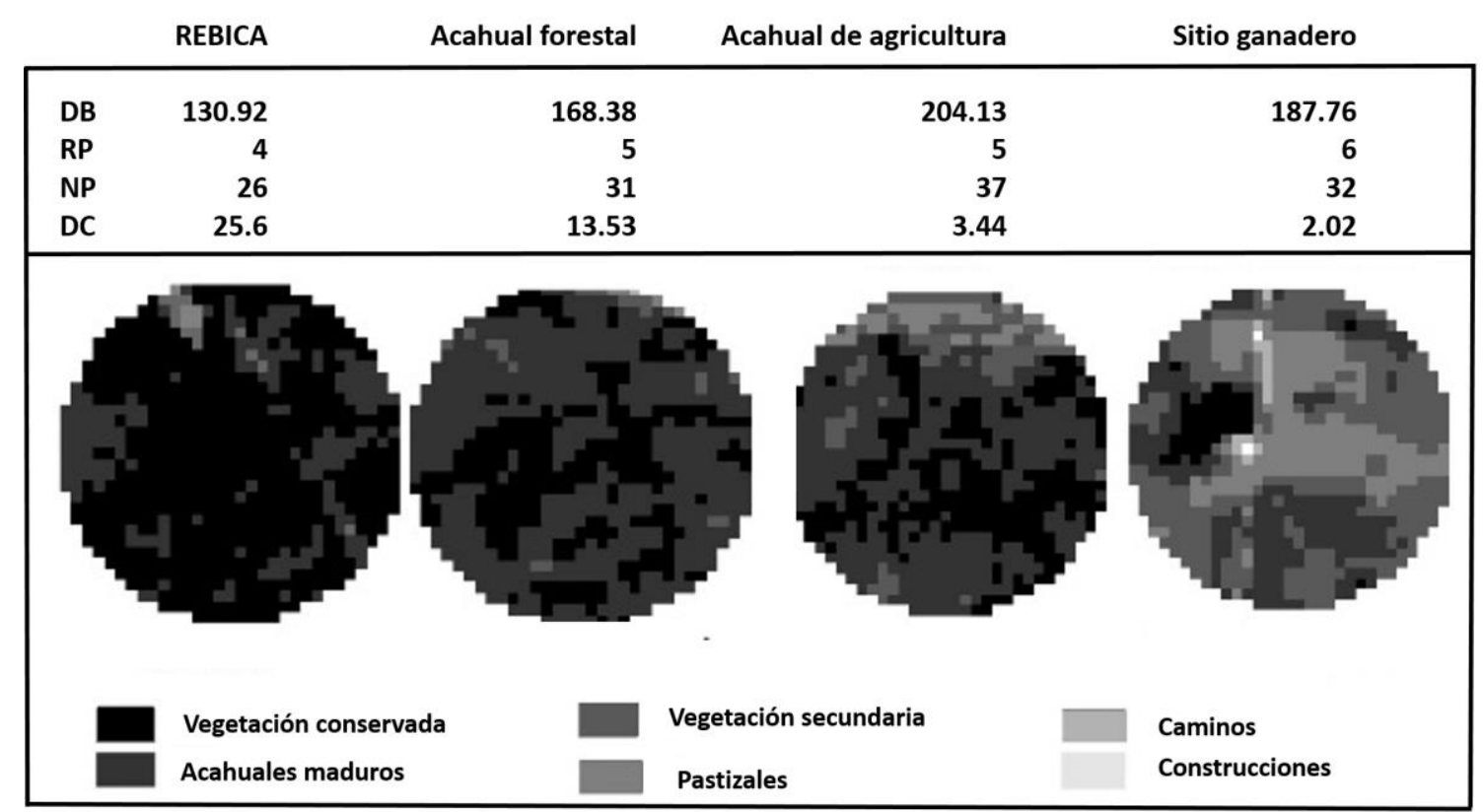

Figura 2. Resultados del análisis de paisaje y cobertura de suelo de cada sitio. Los círculos en escala de grises representan la superficie de cada sitio y cada tono es un tipo de cobertura. DB (Densidad de borde), RP (Riqueza de parches), NP (Número de parches), DC (Distancia a la comunidad en km).

Fueron capturados 415 individuos (nueve de ellos recapturas) representados por siete órdenes, 22 familias y 82 especies. El $42.61 \%$ de las aves capturadas fueron migratorias neotropicales mientras que el $55.41 \%$ fueron aves residentes; el porcentaje restante fueron aves transitorias. La clasificación taxonómica, estacional, y por gremio alimenticio se muestra en el Apéndice 1. El sitio con mayor riqueza de especies fue 
el ganadero $\left(D_{0}=36\right)$ y su comunidad estuvo dominada por una menor cantidad de especies $\left(D_{2}=3.88\right)$ La mayor diversidad observada estuvo en el acahual de agricultura $\left(D_{1}=26.59\right)$, mientras que la mayor diversidad estimada la tuvo la REBICA $\left(\mathrm{D}_{1}=36.38\right)$. Los valores de riqueza $\left(\mathrm{D}_{0}\right)$, diversidad $\left(\mathrm{D}_{1}\right)$ y dominancia $\left(\mathrm{D}_{2}\right)$ de los cuatro sitios de muestreo se muestran en la figura 3.
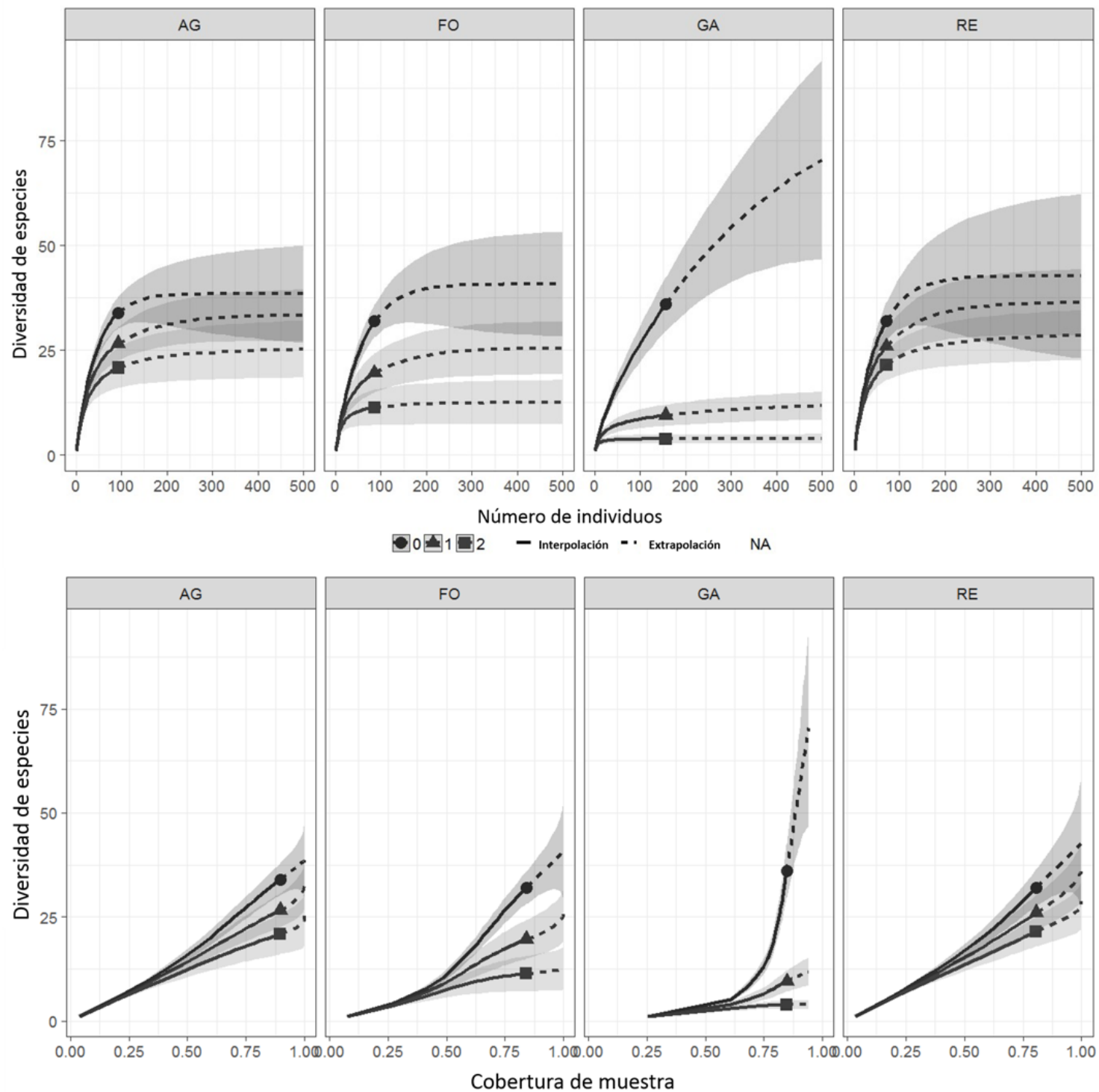

- 01 1 2 - Interpolación -. Extrapolación NA

Figura 3. Riqueza (0), diversidad (1) y especies dominantes (2) en cada sitio de muestreo. La parte superior de la figura representa la diversidad de especies con relación al número de individuos capturados, mientras que la parte inferior representa la diversidad de especies con relación a la cobertura de muestra en cada sitio de muestreo. Se observa en la figura una diferencia en la magnitud de las comunidades de más del doble entre todos los sitios y el sitio ganadero, siendo los órdenes 0 y 1 (riqueza y diversidad, respectivamente) en los que se puede apreciar de manera más significativa esta diferencia, contrastantemente, se observa que la comunidad del sitio ganadero es dominada por una menor cantidad de especies (orden 2); AG (Acahual de agricultura); FO (Acahual forestal); GA

(Sitio ganadero); RE (Reserva de la Biósfera de Calakmul).

Encontramos una mayor equidad en la comunidad de aves en la REBICA (0.82), seguida por el acahual de agricultura (0.78), el acahual forestal (0.59), y la menor la presentó el sitio ganadero (0.40). La 
diversidad beta de la comunidad fue $\beta \mathrm{w}=1.44$. Los sitios con mayor similitud respecto al índice de Jaccard fueron ambos acahuales (0.40) mientras que los menos similares fueron la REBICA y el sitio ganadero (0.15); la similitud fue relativamente media entre la REBICA y el acahual forestal (0.36) y de la REBICA con el acahual de agricultura (0.24).

La abundancia de las especies no presentó una distribución normal $(\mathrm{W}=0.66, p<0.001)$. Se encontraron diferencias significativas en relación con la abundancia de aves migratorias neotropicales en los meses de enero y junio (Kruskal Wallis; $\mathrm{X}^{2}=13.88 \mathrm{df}=4, p=0.007$ ). También existieron diferencias entre los sitios con relación al gremio de los semilleros $\left(\mathrm{X}^{2}=15.22, \mathrm{df}=3, p=0.001\right)$ entre el sitio ganadero y acahual forestal $(p=0.024)$, y sitio ganadero y la REBICA $(p=0.001)$; también entre los sitios con relación al gremio de los insectívoros $\left(\mathrm{X}^{2}=8.47, \mathrm{df}=3, p=0.037\right)$ entre el acahual forestal y la REBICA. Fueron identificados 32 singletons, seis en la REBICA, cuatro en el acahual forestal, cinco en el acahual de agricultura y 17 en el sitio ganadero (Apéndice 1).

El CCA con las variables DISC+ACM+SINV presentó significancia estadística ( $p=0.001)$ y explicó alrededor del $23 \%$ de la variación total. Las variables individualmente fueron también significativas, DISC $(\mathrm{F}=1.74, p=0.001), \operatorname{ACM}(\mathrm{F}=1.57, p=0.001)$ y $\operatorname{SINV}(\mathrm{F}=1.41, p=0.019$ (Fig. 4). Conforme a los GLM el uso de suelo de cada sitio influyó en la abundancia de las especies de aves (Cuadro 1).

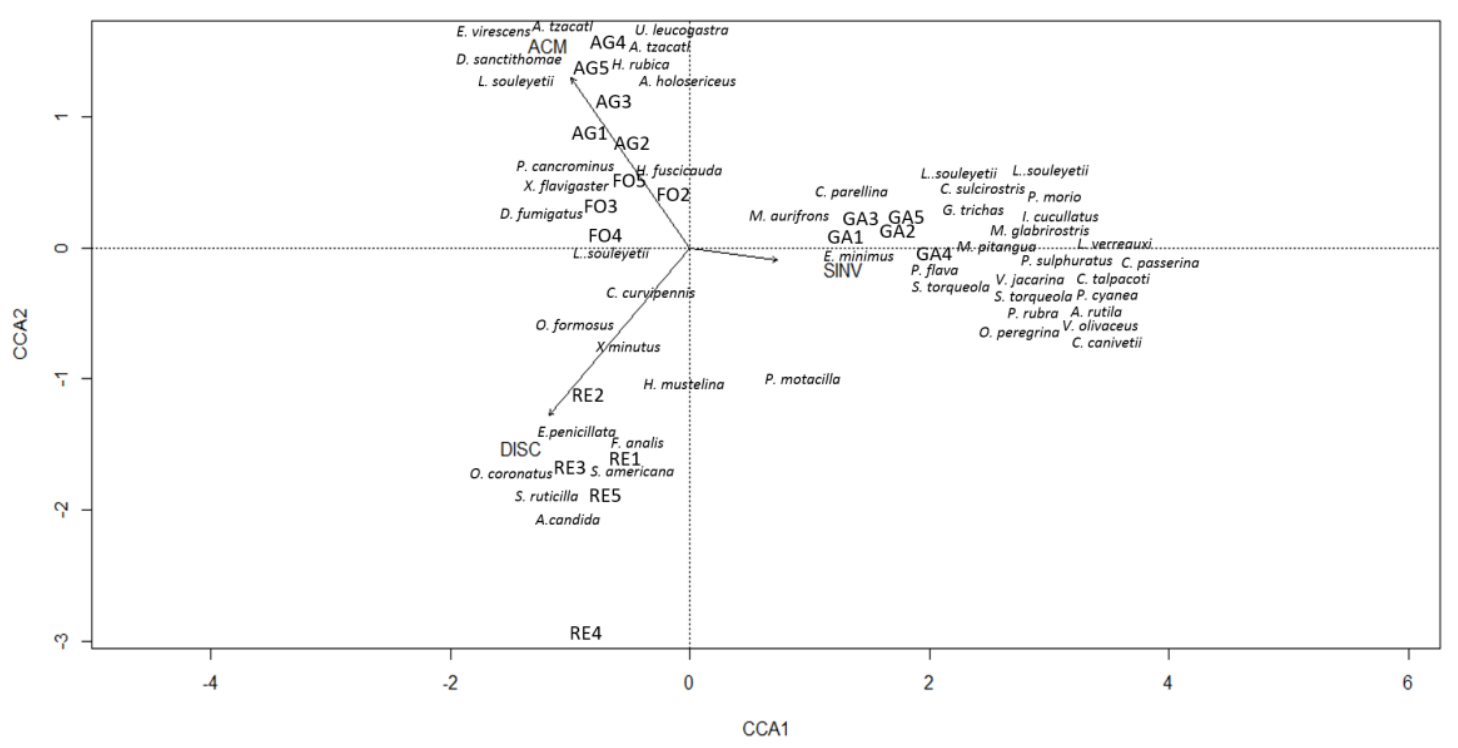

Figura 4. Ordenación gráfica de los sitios muestreados. Se muestran las especies de aves más representativas en los sitios. Los acrónimos con números consecutivos del uno al cinco, pertenecen al número de repeticiones para cada una de las condiciones, en las cuales se puede apreciar la asociación de las especies a cada condición de uso; AG (Acahual de agricultura); FO (Acahual forestal); GA (Sitio ganadero); RE (Reserva de la Biósfera de Calakmul), DISC (Distancia en km a Nuevo Conhuas), SINV (Sin vegetación), ACM (Acahuales maduros).

\section{DISCUSIÓN}

El uso de suelo tiene un efecto sobre la diversidad de las comunidades de aves, lo que fue evidente en los sitios muestreados de nuestro estudio. Nuestros resultados sugieren que los paisajes más heterogéneos y fragmentados favorecen la riqueza de aves, ya que el acahual de agricultura y el sitio ganadero tuvieron mayor heterogeneidad de cobertura, mayor fragmentación y presentaron una mayor riqueza de especies. La 
mayor riqueza de especies de aves observada fue en el sitio ganadero, lo que coincide con lo descrito por Ugalde et al. (2010) y Bojorges (2005) quienes encontraron resultados similares en un bosque templado en el Estado de México y una selva mediana en Veracruz, respectivamente. Sin embargo, muchas de las especies encontradas en el sitio ganadero, como Columbina passerina (Linnaeus, 1758), Columbina tlapacoti, Turdus grayi (Bonaparte, 1838), Volatinia jacarina (Linnaeus, 1766), Cyanocompsa parellina (Linnaeus, 1766) y Passerina cyanea (Linnaeus, 1766) son consideradas como especies generalistas. Esto coincide con lo reportado con Rangel-Salazar et al. (2009), quienes realizaron una comparación entre sitios bajo distintas condiciones de perturbación en un bosque templado en Chiapas y encontraron que el sitio más perturbado presentó mayor riqueza y menor diversidad de aves. Esto también se relaciona con lo descrito por Fahrig $(2003,2017)$ y De Camargo et al. (2018) quienes argumentan que la riqueza de aves se ve afectada por la pérdida de hábitat y no por la fragmentación. Por otro lado, en la REBICA, que tuvo la mayor superficie de vegetación conservada, menor presión por actividades humanas, y la mayor diversidad estimada, registramos especies como Onychorhynchus coronatus (Muller, 1776) (en peligro de extinción), Formicarius analis (d'Orbigny \& Lafresnaye, 1837), Eucometis penicillata (Spix, 1825) (bajo protección especial), Amazilia candida y Chloroceryle aenea, que son especies que han sido reportadas como especialistas y se encuentran enlistadas bajo alguna categoría de protección (SEMARNAT, 2010), que además son consideradas como especies de interior de selva y para las que la perturbación puede ser un factor desfavorable. Hanski (2015) encuentra que sitios con menor perturbación y fragmentación tienden a favorecer la diversidad por lo que, aunque la fragmentación del hábitat puede parecer que incrementa la riqueza, se pierde diversidad y especies especialistas. La ausencia de las especies anteriormente mencionadas en los otros sitios puede estar relacionada con la intensificación de las actividades humanas, situación que puede afectar a cierta clase de aves (Smith et al., 2001). En este sentido, la estructura de la vegetación, como reflejo del uso de suelo, puede determinar la composición de las comunidades de aves (Ugalde et al., 2009).

Cuadro 1. Resultados de los coeficientes de los GLM para las especies de aves y las familias en los que se muestran los grados de libertad (Gl), Devianza (Dev), Significancia del modelo y de las variables utilizadas en cada modelo $(p)$ y la suma de la información del criterio de Akaike (AIC) para cada modelo. Los valores de las variables de cada modelo fueron obtenidos por medio del análisis del paisaje. SP (Especies), VEC (Vegetación conservada), ACM (Acahuales maduros), SINV (Sin vegetación), FAM (Familias de aves).

\begin{tabular}{lcccc}
\hline Modelo & Gl & Dev & $\boldsymbol{p}$ & AIC \\
\hline GLM para especies de aves & & & & \\
\hline SP VEC+ACM+SINV & 19 & 373.1 & 0.001 & \\
VEC & 18 & 186.20 & 0.002 & 1923.124 \\
ACM & 17 & 117.11 & 0.006 & \\
SINV & 16 & 69.83 & 0.01 & \\
\hline GLM para familias de aves & & & & \\
\hline FAM VEC+ACM+SINV & 19 & 114.47 & 0.001 & 695.582 \\
VEC & 18 & 52.17 & 0.002 & \\
ACM & 17 & 40.46 & 0.005 & \\
SINV & 16 & 21.84 & 0.043 & \\
\hline
\end{tabular}

La riqueza de especies de aves observada en este estudio equivale al $20.3 \%$ descrita por González et al. (2016), quienes reportan 403 especies para la REBICA. La diferencia en la magnitud de la diversidad de las comunidades de aves es muy grande entre los sitios, siendo esta magnitud más del doble en los otros sitios en comparación con el sitio ganadero. Los valores de equidad en las comunidades muestran el mismo patrón, siendo el sitio ganadero el que tiene el valor más bajo, situación que resulta de la alta abundancia de 
pocas especies en este sitio. Tal como encontramos en nuestro estudio, la comunidad de aves en el sitio ganadero tiende a estabilizarse más rápidamente al alcanzar un mismo nivel de cobertura de muestra.

La similitud entre los sitios de la REBICA y los acahuales, coincide con los hallazgos de Smith et al. (2001), quienes mencionan que especies de interior se pueden encontrar en sitios en regeneración; se ha reportado también para bosques de latifoliadas (Vázquez et al., 2017) dónde las áreas en regeneración se recuperan rápidamente y se pueden encontrar en estos sitios especies de aves de selvas maduras o de sitios con mayor estado de conservación. La similitud de las especies entre los acahuales y la REBICA puede significar una transición tendiente a la similitud de las comunidades de aves conforme madura la selva, lo que pudiera influir en la recuperación de las poblaciones de algunas especies. Por el contrario, la mayor diferencia fue entre la REBICA y el sitio ganadero, que actualmente tiene una presión permanente por actividades humanas, lo que explica esta disimilitud en la composición de especies.

Respecto a la abundancia de aves migratorias neotropicales, solamente encontramos diferencias con relación a los meses de muestreo y no en cuanto al uso de suelo. Lo que encontramos no coincide con lo descrito por Robbins et al. (1989) y González et al. (2016), quienes mencionan que la deforestación y el efecto acumulativo del uso de suelo pueden afectar al grupo de aves migratorias. Con base en nuestros resultados, proponemos que las aves migratorias utilizan sitios con distinto uso de suelo y estados de perturbación en los sitios donde pasan el invierno o usan durante la migración más al sur. Se requiere un mayor esfuerzo de muestreo para corroborar esta situación.

Al comparar las abundancias de los gremios encontramos diferencias significativas, con lo que sugerimos que existe una preferencia de uso de los distintos sitios por parte de los gremios, lo cual ha sido descrito en otras investigaciones (Petit \& Petit, 2003; Wilson et al., 1996). Pueden existir tendencias en la presencia de gremios en sitios con distinto uso de suelo y cobertura de vegetación, por ejemplo, los semilleros, que en este estudio estuvieron representados por especies generalistas, pueden ser favorecidos en áreas abiertas en las que las presiones antropogénicas son constantes, mientras que otros gremios la respuesta puede ser inversa.

En el sitio ganadero encontramos una mayor abundancia de especies que se registraron en una sola o en dos unidades de muestreo ( $\mathrm{f} 1$ y f2); sin embargo, la mayoría de estas aves son consideradas generalistas, de espacios abiertos y que se caracterizan por estar presentes en sitios perturbados o bajo presión de actividades humanas. Wall et al. (2012), describieron que los paisajes agroforestales son importantes para este grupo de aves, situación que pudiera estar ocurriendo en este sitio.

De manera general, el uso de suelo determinó la composición de la comunidad de aves, y aunque el CCA explicó solamente el $23 \%$ de la variación total, el resultado refuerza lo encontrado con el índice de Jaccard. La distancia entre los acahuales y la presencia e identidad de las especies de aves en ellos muestra una tendencia en la composición de la comunidad. En este sentido, la estructura de la vegetación en los sitios puede determinar la composición de las comunidades de aves (Ugalde et al., 2009), y la variación no explicada por el CCA, puede estar relacionada a condiciones de hábitat y microhábitat, como la estructura, identidad o abundancia de las especies vegetales presentes en cada sitio.

De acuerdo con nuestros resultados en los modelos (GLM) las familias Cardinalidae, Columbidae y Thraupidae son favorecidas por sitios sin vegetación, mientras que la familia Furnariidae, principalmente representada por los trepatroncos, se vio influenciada por los acahuales y la vegetación conservada en la REBICA. Lo anterior remarca la importancia de los acahuales como sitios de transición hacia selvas maduras.

La familia Thraupidae, cuya única especie fue E. penicilata, resultó favorecida por la vegetación conservada y estuvo presente solamente en la REBICA, aunque tuvo una baja abundancia. A nivel de 
especie, Dendrocincla homochroa (Sclater, 1859), Habia fuscicauda (Cabanis, 1861) y Arremonops rufivirgatus (Lawrence, 1851), pertenecientes a las familias Furniriidae, Cardinalidae y Thraupidae, respectivamente, fueron fuertemente influenciadas por la vegetación, lo que coincide con los resultados encontrados para las familias. Un mayor esfuerzo de muestreo (tiempo y número de redes) podría incrementar la abundancia de algunas especies, lo cual ayudaría a realizar estimaciones más acertadas. Es necesario analizar la influencia de la cobertura de suelo y heterogeneidad de paisaje sobre especies particulares ya que distintas especies pueden responder de diferente manera a los cambios de cobertura, fragmentación y cantidad de hábitat disponible (De Camargo et al., 2018). En este sentido podemos pensar que no solamente las condiciones de uso de suelo favorecen o perjudican la diversidad de aves, sino pueden influenciar la composición de sus comunidades.

\section{CONCLUSIONES}

La diversidad de las comunidades de aves fue favorecida por sitios con mayor cobertura de vegetación conservada y menos cobertura de suelo asociada a actividades antropogénicas. La similitud de la composición de aves en sitios con coberturas similares demuestra la importancia e influencia del uso de suelo y vegetación presente en la composición de las comunidades de aves.

El tipo de uso de suelo es un factor importante en la composición de las comunidades de aves y sitios con uso de suelo tendientes a la regeneración o conservación, pueden ser de importancia para la conservación de la diversidad de aves. Creemos que futuros estudios se deben de focalizar en el impacto particular y la influencia del uso de suelo sobre especies que han sido reportadas como amenazadas, tanto residentes como migratorias neotropicales. Ya que los acahuales presentaron una similitud mayor con la REBICA creemos que deben de realizarse esfuerzos para su manejo y conservación que sean tendientes a la transformación gradual en selvas maduras y sitios con mayor cobertura de vegetación conservada ya que esto favorece la diversidad de las comunidades de aves.

AgradeCimientos. Al Consejo Nacional de Ciencia y Tecnología por la beca ( $\mathrm{N}^{\circ}$. 597639) otorgada al primer autor para la realización de este estudio. A las autoridades del ejido Nuevo Conhuas por las facilidades otorgadas para trabajar en el ejido y sus sitios. A las personas involucradas en el trabajo de campo. A la dirección de la Reserva de la Biósfera de Calakmul por la orientación y facilidades para realizar este estudio. A los revisores, quienes ayudaron a mejorar este documento.

\section{LITERATURA CITADA}

Anders, A. D., Faaborg, J., Thompson, F. R. (1998) Post-fleeding dispersal, habitat use, and home-range size of juvenile wood thrushes. The Auk, 115, 349-358.

http://doi.org/10.2307/4089193

Arizmendi, M. C., Berlanga, H. (2014) Colibríes de México y Norte América. CONABIO. México, 160 pp.

Audubon. Guide to North American Birds. Disponible en: http://www.audubon.org/bird-guide (último acceso en febrero 2018).

Ausprey, I. J., Rodewald, A. D. (2013) Post-fledging dispersal timing and natal range size of two songbird species in an urbanizing landscape. The Condor, 115, 102-114. http://doi.org/10.1525/cond.2013.110176

Berlanga, H., Gómez de Silva, H., Vargas, V. M., Rodríguez, V., Sánchez, L. A., Ortega, R., Calderón, R. (2017) Aves de México: Lista actualizada de especies y nombres comunes. Disponible en: 
http://www.biodiversidad.gob.mx/especies/scripts_aves/docs/lista_actualizada_aos_2017.pdf. (último acceso en febrero 2018).

Bojorges, J. C., López L. (2005) Riqueza y diversidad de aves en una selva mediana subperenifolia en el centro de Veracruz. México. Acta Zoológica Mexicana (nueva serie), 21, 1-20.

Braak, C. J. F. (1987) The analysis of vegetation-environment relationships by canonical correspondence analysis. Vegetatio, 69, 69-77.

Calderón, P. J., Moreno, C. E., Zuria, I. (2012) La diversidad beta: medio siglo de avances. Revista Mexicana de Biodiversidad, 83, 879-891. http://dx.doi.org/10.7550/rmb.25510

Céspedes, F., Moreno, S. (2010) Estimación del valor de la pérdida de recurso forestal y su relación con la reforestación en las entidades federativas de México. Investigación Ambiental, 2, 5-13.

Chao, A., Gotelli, N. J., Hsieh, T. C., Sander, E. L., Ma, K. H., Colwell, R. K., Ellison, A. M. (2014) Rarefaction and extrapolation with Hill numbers: a framework for sampling and estimation in species diversity studies. Ecological Monographs, 84, 45-67. http://doi.org/10.1890/13-0133.1

Chowdhury, R. R. (2006) Landscape change in the Calakmul Biosphere Reserve, Mexico: Modeling the driving forces of smallholder deforestation in land parcels. Applied Geography, 26, 129-152. http://doi.org/10.1016/j.apgeog.2005.11.004

Colwell, R. K. (2013) EstimateS: Statistical estimation of species richness and shared species from samples. Versión 9. http://viceroy.eeb.uconn.edu/estimates.

Connell, J. H. (1978) Diversity in tropical rain forests and coral reefs: High diversity of trees and coral is maintained only in a non equilibrium state. Science, 199, 1302-1310. http://doi.org/10.1126/science.199.4335.1302

Cox, G. W. (2010) Bird migration and global change. Island Press, United states of América, 304 pp.

De Camargo, R. X., Boucher, B., Currie, D. J. (2018) At the landscape level, birds respond strongly to habitat amount but weakly to fragmentation. Diversity and Distributions, 24, 629-639. http://doi.org/10.1111/ddi.12706

Ellis, E. A., Romero, J. A., Hernández, I. U. (2017) Deforestation processes in the state of Quintana Roo, Mexico: The Role of Land Use and Community Forestry. Tropical Conservation Science, 10, 1-12. http://doi.org/10.1177/1940082917697259

Fahrig, L. (2003) Effects of habitat fragmentation on biodiversity. Annual Review of Ecology and Systematics, 34, 487-515. http://doi.org/10.1146/annurev.ecolsys.34.011802.132419

Fahrig, L. (2017) Ecological responses to habitat fragmentation Per Se. Annual Review of Ecology and Systematics, 48, 1-23. http://doi.org/10.1146/annurev-ecolsys-110316-022612

Fox, J. W. (2013) The intermediate disturbance hypothesis should be abandoned. Trends in Ecology \& Evolution, 28, 85-92. http://doi.org/10.1016/j.tree.2012.08.014

García, E. (1973) Modificaciones al Sistema de Clasificación Climática de Köppen (Para Adaptarlo a las Condiciones de la República Mexicana). 2a. Ed., Universidad Nacional Autónoma de México, México, D.F.

González, M., Martínez, E., Rangel, J. L. (2016) Actualización del inventario de la avifauna de la Reserva de la Biósfera de Calakmul, península de Yucatán, México: abundancia, estacionalidad y categoría de conservación. Huitzil, 17, 54-106.

Granados, D., López, G. F., Trujillo, E. (1999) La milpa en la zona maya de Quintana Roo. Revista de Geografía Agrícola, 28, 57-72.

Greenberg, R., Bichier, P., Sterling, J. (1997) Bird Populations in rustic and planted shade coffee plantations of eastern Chiapas, México. Biotropica, 29, 501-514. http://doi.org/10.1111/j.1744-7429.1997.tb00044.x

Hanski, I. (2015) Habitat fragmentation and species richness. Journal of Biogeography, 42, 989-994. 
http://doi.org/10.1111/jbi.12478

INEGI (Instituto Nacional de Estadística y Geografía) (2015) Conociendo México. México, 30 pp.

Jirinec, V., Varian, C. P., Smith, C. J., Leu, M. (2016) Mismatch between diurnal home ranges and roosting areas in the Wood Thrush (Hylocichla mustelina): Possible role of habitat and breeding stage. The Auk, 133, 1-12.

http://doi.org/10.1642/AUK-15-76.1

Jost, L. (2010) The relation between evenness and diversity. Diversity, 2, 207-232. http://doi.org/10.3390/d2020207

Kaufman, K. (2005) Guía de campo para las aves de Norteamérica. Houghton Mifflin, United States of América, 392 pp.

Kendall, W. L., Converse, S. J., Doherty, P. F., Naughton, M. B., Anders, A., Hines, J. E., Flint, E. (2009) Sampling design considerations for demographic studies: A case of colonial seabirds. Ecological Applications, 19, 55-68. http://doi.org/10.1890/07-1072.1

Legendre, P., Gallagher, E. D. (2001) Ecologically meaningful transformations for ordination of species data. Oecología, 129, 271-280. http://doi.org/10.1007/s004420100716

Levy, S. I., Aguirre, R. J. (2000) El aprovechamiento agrícola intensivo de los Hubchés (Acahuales o comunidades secundarias) de Yucatán. Revista Geográfica, 128, 79-103.

MacKinnon, B. H. (2013) Sal a pajarear Yucatán (guía de aves). La Vaca Independiente, Mexico, 287 pp.

Martínez, E., Galindo, C. (2002) La vegetación de Calakmul, Campeche, México: clasificación, descripción y distribución. Boletín la Sociedad Botánica México, 71, 7-32.

McCune, B., Grace, J. B. (2002) Analysis of Ecological Communities. MjM Software, United States of America, 304 pp. http://doi.org/10.1016/S0022-0981(03)00091-1

McGarigal, K., Cushman, S. A., Ene, E. (2012) FRAGSTATS v4: Spatial Pattern Analysis Program for Categorical and Continuous Maps. Disponible http://www.umass.edu/landeco/research/fragstats/fragstats.html

Miranda, F. (1958) Rasgos fisiográficos de interés para los estudios biológicos. Pp: 161-163. En: E. Beltrán (Eds.). Los Recursos naturales del sureste y su aprovechamiento. Tomo II. Instituto Mexicano de Recursos Renovables. México, D.F.

Moreno, C. E., Barragán, F., Pineda, E., Pavón, N. P. (2010) Reanálisis de la diversidad alfa: alternativas para interpretar y comparar información sobre comunidades ecológicas. Revista Mexicana de Biodiversidad, 82, 1249-1261.

Naturalista. CONABIO. Disponible en: http://www.naturalista.mx (ultimo acceso en febrero 2018).

Navarro, S. A. G., Rebón, F. M., Gordillo, A., Towsend, A., Berlanga, H., Sánchez, L. A. (2014) Biodiversidad de aves en México. Revista Mexicana de Biodiversidad, 85, 476-495. http://dx.doi.org/10.7550/rmb.41882

Neotropical Birds Online. Cornell Lab of Ornithology. Disponible en: http://neotropical.birds.cornell.edu/Species (último acceso en febrero 2018).

Neri, M. (2007) Ficha técnica de Eucometis penicillata. En: P. Escalante-Pliego (Eds.). "Fichas sobre las especies de Aves incluidas en el Proyecto de Norma Oficial Mexicana PROYNOM-ECOL-2000. Parte 2". Instituto de Biología, UNAM, México, D.F.

Newton, I. (2008) The migration ecology of birds. Elsevier, Oxford, United Kingdom, 984 pp.

Oksanen, J., Blanchet, F. G., Friendly, M., Kindt, P. R., Legendre, P., McGlinn, D., Minchin, P. R., O'Hara, R. B., Simpson, G. L., Solymos, P., Stevens, M. H. H., Szoecs, E., Wagner, H. (2018) Vegan: Community Ecology Package. $\mathrm{R}$ package version 2.4-6. http://CRAN.Rproject.org/package $=$ vegan

Perlo, B. V. (2006) Birds of Mexico and Central America. Princeton, Princeton University Press, United States of America, $336 \mathrm{pp}$.

Petit, L. J., Petit, D. R. (2003) Evaluating the importance of human-modified lands for neotropical bird conservation. Conservation Biology, 17, 687-694. 
Rangel-Salazar, J. L, Enríquez, P. L., Sántiz, E. C. (2009) Variación de la diversidad de aves de sotobosque en el Parque Nacional Lagos de Montebello, Chiapas, México. Acta Zoológica Mexicana (nueva serie), 25, 479-495.

Ralph, C. J., Dunn, E. H., Peach, W. J., Handel, C. M. (2001) Recommendations for the use of mist nets for inventory and monitoring of bird populations. Studies in Avian Biology, 29, 187-196.

Robbins, C. S., Sauer, J. R., Greenberg, R. S., Droege, S. (1989) Population declines in North American birds that migrate to the neotropics. Proceedings of the National Academy of Sciences, 86, 7658 7662 .

RStudio Team (2016) RStudio: Integrated Development for R. RStudio, Inc., Boston, MA. Disponible en: http://www.rstudio.com/

Sekercioglu, C. H. (2006) Ecological significance of bird populations. Pp: 15-34. En: J. Del Hoyo, A. Elliot, D. Christie (Eds.). Handbook of the Birds of the World. BirdLife International, España, Barcelona.

SEMARNAP (Secretaría de Medio Ambiente, Recursos Naturales y Pesca) (1999) Programa de Manejo de la Reserva de la Biósfera de Calakmul. Instituto Nacional de Ecología, México, 270 pp.

SEMARNAT (Secretaría de Medio Ambiente y Recursos Naturales) (2010) Norma Oficial Mexicana nom-059-semarnat-2010. Diario Oficial de la Federación 30 de diciembre de 2010, Segunda Sección. México, D.F., 77 pp.

Sibley, D. A. (2014) The Sibley guide to birds. 2nd ed. United States of America, 598 pp.

Smith, A. L., Ortiz, J., Robertson, R. J. (2001) Distribution patterns of migrant and resident birds in successional forests of the Yucatan Peninsula, Mexico. Biotropica, 33, 153-170. http://doi.org/10.1111/j.1744-7429.2001.tb00165.x

Ugalde, S., Valdez, J. I., Ramírez, G., Alcántara, J. L., Velázquez, J. (2009) Distribución vertical de aves en un bosque templado con diferentes niveles de perturbación. Madera y Bosques, 15, 5-26.

Ugalde, S., Alcántara, J. L., Valdez, J. I., Ramírez, G., Velázquez, J., Tarángo, L. A. (2010) Riqueza, abundancia y diversidad de aves en un bosque templado con diferentes condiciones de perturbación. Agrociencia, 44, 159-169.

USGS (2017) United States Geological Service. Earth Explorer. Disponible en: http://www.usgs.gov (último acceso en septiembre 2017).

Van, S. A., Philpott, S. M., Greenberg, R., Bichier, P., Barber, N. A., Mooney, K. A., Gruner, D. S. (2008) Birds as predators in tropical agroforestry systems. Ecology, 89, 928-934. http://doi.org/10.1890/06-1976.1

Vázquez, L. D., Arizmendi, M., Godínez, H. O., Navarro, A. G. (2017) Directional effects of biotic homogenization of bird communities in Mexican seasonal forests. The Condor, 119, 275-288. http://doi.org/10.1650/CONDOR-16-116.1

Wall, H. V. D., Peña, B., Arriaga, S. L., Hernández, S. (2012) Species, functional groups, and habitat preferences of birds in five agroforestry classes in Tabasco, Mexico. Wilson Journal of Ornithology, $124,558-571$. http://doi.org/10.1676/10-111.1

Wang, Y., Naumann, U., Wright, S., Warton, D. I. (2012) mvabund: an R package for model-based analysis of multivariate data. Methods in Ecology and Evolution, 3, 471-474. http://doi.org/10.1111/j.2041-210X.2012.00190.x

Warkentin, I. G., Greenberg, R., Ortiz, J. S. (1995) Songbird use of gallery woodlands in recently cleared and older settled landscapes of the Selva Lacandona, Chiapas, Mexico. Conservation Biology, 9, 1095-1106.

Wilson, J. D., Taylor, R., Miurhead, L. B. (1996) Field use by farmland birds in winter: an analysis of field type preferences using resampling methods. Bird Study, 43, 320-332. http://doi.org/10.1080/00063659609461025 
Apéndice 1. Clasificación taxonómica conforme al American Ornithological Society (Berlanga et al., 2019), número de individuos, estacionalidad (EST) y gremio alimenticio (GAL) de las aves capturadas en cuatro sitios en el ejido Nuevo Conhuas, Calakmul, Campeche durante febrero a junio de 2017. Las especies con asterisco (*) representan los individuos recapturados durante el muestreo.

\begin{tabular}{|c|c|c|c|c|c|c|c|c|c|}
\hline \multirow{2}{*}{ Orden } & \multirow{2}{*}{ Familia } & \multirow{2}{*}{ Nombre científico } & \multirow{2}{*}{ Nombre común } & \multicolumn{4}{|c|}{$\begin{array}{c}\text { Sitios de captura y número } \\
\text { de individuos }\end{array}$} & \multirow[t]{2}{*}{ EST } & \multirow[t]{2}{*}{ GAL } \\
\hline & & & & $\begin{array}{l}\text { RES } \\
(74)\end{array}$ & $\begin{array}{c}\text { FOR } \\
(89)\end{array}$ & $\begin{array}{c}A G R \\
(98)\end{array}$ & $\begin{array}{l}\text { GAN } \\
(154)\end{array}$ & & \\
\hline \multirow[t]{7}{*}{ Apodiformes } & Trochilidae & Amazilia candida & Colibrí cándido & 1 & & & & $\mathrm{R}$ & $\mathrm{N}$ \\
\hline & & Amazilia rutila & Colibrí canela & & 1 & & & $\mathrm{R}$ & $\mathrm{N}$ \\
\hline & & Amazilia tzacatl & Colibrí cola rojiza & & & 2 & & $\mathrm{R}$ & $\mathrm{N}$ \\
\hline & & Amazilia yucatanensis* & Colibrí yucateco & 1 & 2 & 3 & 3 & $\mathrm{R}$ & $\mathrm{N}$ \\
\hline & & Archilochus colubris & Colibrí garganta rubí & & & & 1 & M & $\mathrm{N}$ \\
\hline & & Campylopterus curvipennis & Fandanguero mexicano & 4 & 4 & 2 & 2 & $\mathrm{R}$ & $\mathrm{N}$ \\
\hline & & Chlorostilbon canivetii & Esmeralda tijereta & & & & 1 & $\mathrm{R}$ & $\mathrm{N}$ \\
\hline \multirow[t]{3}{*}{ Columbiformes } & Columbidae & Columbina passerina & Tórtola coquita & & & & 8 & $\mathrm{R}$ & $\mathrm{S}$ \\
\hline & & Columbina talpacoti & Tórtola rojiza & & & & 1 & $\mathrm{R}$ & $\mathrm{S}$ \\
\hline & & Leptotila verreauxi & Paloma arroyera & & & & 2 & $\mathrm{R}$ & $\mathrm{S}$ \\
\hline Coraciiformes & Alcedinidae & Chloroceryle aenea & Martín pescador enano & 1 & & & & $\mathrm{R}$ & $\mathrm{O}$ \\
\hline Cuculiformes & Cuculidae & Crotophaga sulcirostris & Garrapatero & & & & 1 & $\mathrm{R}$ & $\mathrm{O}$ \\
\hline \multirow[t]{13}{*}{ Passeriformes } & Cardinalidae & Cyanocompsa parellina & Colorín azul negro & & 2 & 3 & 13 & $\mathrm{R}$ & $\mathrm{S}$ \\
\hline & & Granatellus sallaei & Granatelo yucateco & & & 2 & & $\mathrm{R}$ & $\mathrm{S}$ \\
\hline & & Habia fuscicauda & Tángara-hormiguera garganta roja & & 10 & 6 & & $\mathrm{R}$ & I \\
\hline & & Habia rubica & Tángara-hormiguera corona roja & & & 3 & & $\mathrm{R}$ & $\mathrm{I}$ \\
\hline & & Passerina ciris & Colorín siete colores & & & & 2 & M & $\mathrm{S}$ \\
\hline & & Passerina cyanea & Colorín azul & & 1 & & 76 & M & $\mathrm{S}$ \\
\hline & & Piranga flava & Tángara encinera & & & 1 & & $\mathrm{R}$ & $\mathrm{I}$ \\
\hline & & Piranga roseogularis & Tángara yucateca & & 2 & 2 & & $\mathrm{R}$ & $\mathrm{O}$ \\
\hline & & Piranga rubra & Tángara roja & & & & 1 & M & $\mathrm{I}$ \\
\hline & Corvidae & Psilorhinus morio & Chara café & & & & 1 & $\mathrm{R}$ & $\mathrm{O}$ \\
\hline & Formicariidae & Formicarius analis & Hormiguero cara negra & 3 & & & & $\mathrm{R}$ & $\mathrm{I}$ \\
\hline & Fringillidae & Euphonia affinis & Eufonia garganta negra & 1 & & & & $R$ & $\mathrm{O}$ \\
\hline & Furnariidae & Dendrocincla anabatina & Trepatroncos sepia & 2 & 2 & 2 & & $\mathrm{R}$ & I \\
\hline
\end{tabular}




\begin{tabular}{|c|c|c|c|c|c|c|c|c|c|}
\hline \multirow{2}{*}{ Orden } & \multirow{2}{*}{ Familia } & \multirow{2}{*}{ Nombre científico } & \multirow{2}{*}{ Nombre común } & \multicolumn{4}{|c|}{$\begin{array}{c}\text { Sitios de captura y número } \\
\text { de individuos }\end{array}$} & \multirow[t]{2}{*}{ EST } & \multirow[t]{2}{*}{ GAL } \\
\hline & & & & $\begin{array}{l}\text { RES } \\
(74)\end{array}$ & $\begin{array}{c}\text { FOR } \\
(89)\end{array}$ & $\begin{array}{c}A G R \\
(98)\end{array}$ & $\begin{array}{l}\text { GAN } \\
(154)\end{array}$ & & \\
\hline & \multirow[t]{6}{*}{ Furnariidae } & Dendrocincla homochroa* & Trepatroncos rojizo & 2 & 21 & 4 & & $\mathrm{R}$ & I \\
\hline & & Dendrocolaptes sanctithomae & Trepatroncos barrado & & & 2 & & $\mathrm{R}$ & I \\
\hline & & Lepidocolaptes souleyetii & Trepatroncos corona rayada & & & 1 & & $\mathrm{R}$ & I \\
\hline & & Sittasomus griseicapillus & Trepatroncos oliváceo & 1 & 7 & 1 & & $\mathrm{R}$ & I \\
\hline & & Xenops minutus & Picolezna liso & 2 & 2 & & & $\mathrm{R}$ & I \\
\hline & & Xiphorhynchus flavigaster* & Trepatroncos bigotudo & 3 & 2 & 9 & & $\mathrm{R}$ & I \\
\hline & \multirow[t]{2}{*}{ Icteridae } & Amblycercus holosericeus & Cacique pico claro & & & 1 & & $\mathrm{R}$ & I \\
\hline & & Icterus cucullatus & Bolsero encapuchado & & & & 1 & $\mathrm{R}$ & I \\
\hline & \multirow[t]{2}{*}{ Mimidae } & Dumetella carolinensis & Maullador gris & 2 & 2 & & & $\mathrm{M}$ & I \\
\hline & & Melanoptila glabrirostris & Maullador negro & & & & 1 & $\mathrm{R}$ & $\mathrm{O}$ \\
\hline & Onychorhynchidae & Onychorhynchus coronatus* & Mosquero real & 4 & & & & $\mathrm{R}$ & I \\
\hline & \multirow[t]{14}{*}{ Parulidae } & Setophaga magnolia & Chipe de magnolia & 2 & 1 & 2 & 1 & $\mathrm{M}$ & I \\
\hline & & Setophaga pensylvanica & Chipe flanco castaño & & & 1 & & $\mathrm{~T}$ & I \\
\hline & & Geothlypis trichas & Mascarita común & & & & 2 & M & I \\
\hline & & Helmitheros vermivorum & Chipe gusanero & 1 & 1 & & & M & I \\
\hline & & Limnothlypis swainsonii & Chipe corona café & 1 & 1 & & & M & I \\
\hline & & Mniotilta varia* & Chipe trepador & 4 & 2 & 3 & & M & I \\
\hline & & Geothlypis formosa & Chipe patilludo & 2 & & 1 & & M & I \\
\hline & & Setophaga americana & Parula norteña & 1 & & & & M & I \\
\hline & & Seiurus aurocapilla & Chipe suelero & 6 & 3 & 2 & 1 & M & I \\
\hline & & Parkesia motacilla & Chipe arroyero & 1 & & & 1 & $\mathrm{~T}$ & I \\
\hline & & Parkesia noveboracensis & Chipe charquero & & 3 & & & M & I \\
\hline & & Setophaga ruticilla & Chipe flameante & 1 & & & & M & I \\
\hline & & Leiothlypis peregrina & Chipe peregrino & & & & 2 & $\mathrm{~T}$ & I \\
\hline & & Setophaga citrina* & Chipe encapuchado & 8 & 3 & 11 & & M & I \\
\hline & Passerellidae & Arremonops rufivirgatus* & Rascador oliváceo & 1 & & 7 & & $\mathrm{R}$ & $\mathrm{S}$ \\
\hline & Thraupidae & Eucometis penicillata & Tángara cabeza gris & 4 & & & & $\mathrm{R}$ & $\mathrm{I}$ \\
\hline
\end{tabular}




\begin{tabular}{|c|c|c|c|c|c|c|c|c|c|}
\hline \multirow{2}{*}{ Orden } & \multirow{2}{*}{ Familia } & \multirow{2}{*}{ Nombre científico } & \multirow{2}{*}{ Nombre común } & \multicolumn{4}{|c|}{$\begin{array}{c}\text { Sitios de captura y número } \\
\text { de individuos }\end{array}$} & \multirow[t]{2}{*}{ EST } & \multirow[t]{2}{*}{ GAL } \\
\hline & & & & $\begin{array}{l}\text { RES } \\
(74)\end{array}$ & $\begin{array}{c}\text { FOR } \\
(89)\end{array}$ & $\begin{array}{c}A G R \\
(98)\end{array}$ & $\begin{array}{l}\text { GAN } \\
(154)\end{array}$ & & \\
\hline & Thraupidae & Volatinia jacarina & Semillero brincador & & & & 13 & $\mathrm{R}$ & $\mathrm{S}$ \\
\hline & & Sporophila torqueola & Semillero de collar & & & & 1 & $\mathrm{R}$ & $\mathrm{S}$ \\
\hline & Tityridae & Pachyramphus aglaiae & Mosquero cabezón & & & 3 & & $\mathrm{R}$ & I \\
\hline & Troglodytidae & Thryothorus ludovicianus & Chivirín de carolina & & 1 & 2 & & $\mathrm{R}$ & I \\
\hline & & Pheugopedius maculipectus & Chivirín moteado & & 1 & 1 & 1 & $\mathrm{R}$ & I \\
\hline & & Uropsila leucogastra & Chivirín vientre blanco & & & 3 & & $\mathrm{R}$ & I \\
\hline & Turdidae & Catharus ustulatus & Zorsal de Swainson & 2 & & & & M & I \\
\hline & & Hylocichla mustelina* & Zorsal maculado & 6 & 2 & & 1 & M & I \\
\hline & & Turdus grayi & Mirlo pardo & 1 & & & 5 & $\mathrm{R}$ & $\mathrm{O}$ \\
\hline & Tyrannidae & Attila spadiceus & Mosquero atila & 2 & 1 & 3 & & $\mathrm{R}$ & I \\
\hline & & Contopus cinereus & Pibí tropical & & 1 & & & $\mathrm{R}$ & I \\
\hline & & Empidonax minimus & Mosquero mínimo & 1 & & 1 & 4 & M & I \\
\hline & & Empidonax virescens & Mosquero verdoso & & & 1 & & $\mathrm{~T}$ & I \\
\hline & & Megarynchus pitangua & Luis pico grueso & & & & 1 & $\mathrm{R}$ & I \\
\hline & & Myiarchus crinitus & Papamoscas viajero & & 1 & & & M & I \\
\hline & & Myiarchus tuberculifer & Papamoscas triste & 1 & & & & $\mathrm{R}$ & I \\
\hline & & Myiarchus tyrannulus & Papamoscas tirano & & & & 1 & $\mathrm{R}$ & I \\
\hline & & Myiarchus yucatanensis & Papamoscas yucateco & & & & 1 & $\mathrm{R}$ & I \\
\hline & & Oncostoma cinereigulare & Mosquerito pico curvo & & & 2 & & $\mathrm{R}$ & I \\
\hline & & Pitangus sulphuratus & Luis bienteveo & & & & 1 & $\mathrm{R}$ & $\mathrm{O}$ \\
\hline & & Platyrinchus cancrominus & Mosquero pico chato & & 2 & 1 & & $\mathrm{R}$ & I \\
\hline & & Rhynchocyclus brevirostris & Mosquero de anteojos & & 1 & & & $\mathrm{R}$ & $\mathrm{O}$ \\
\hline & & Tolmomyias sulphurescens & Mosquero ojo banco & 2 & 1 & 3 & 1 & $\mathrm{R}$ & I \\
\hline & Vireonidae & Vireo flavifrons & Vireo garganta amarilla & & 2 & & & $\mathrm{M}$ & I \\
\hline & & Vireo griseus & Vireo ojo blanco & & 2 & 6 & & M & I \\
\hline & & Vireo olivaceus & Vireo ojo rojo & & & & 1 & $\mathrm{~T}$ & I \\
\hline Piciformes & Picidae & Melanerpes aurifrons & Carpintero cheje & & 1 & & 1 & $\mathrm{R}$ & $\mathrm{O}$ \\
\hline
\end{tabular}




\begin{tabular}{|c|c|c|c|c|c|c|c|c|c|}
\hline \multirow{2}{*}{ Orden } & \multirow{2}{*}{ Familia } & \multirow{2}{*}{ Nombre científico } & \multirow{2}{*}{ Nombre común } & \multicolumn{4}{|c|}{$\begin{array}{c}\text { Sitios de captura y número } \\
\text { de individuos }\end{array}$} & \multirow[t]{2}{*}{ EST } & \multirow[t]{2}{*}{ GAL } \\
\hline & & & & $\begin{array}{c}\text { RES } \\
\text { (74) }\end{array}$ & $\begin{array}{c}\text { FOR } \\
(89)\end{array}$ & $\begin{array}{c}A G R \\
(98)\end{array}$ & $\begin{array}{l}\text { GAN } \\
(154)\end{array}$ & & \\
\hline & Picidae & Dryobates fumigatus & Carpintero café & & 1 & & & $\mathrm{R}$ & $\mathrm{I}$ \\
\hline & Ramphastidae & Pteroglossus torquatus & Aracari & & & 1 & & $\mathrm{R}$ & $\mathrm{O}$ \\
\hline Psittaciformes & Psittacidae & Eupsittula nana & Perico pecho sucio & & & & 1 & $\mathrm{R}$ & $\mathrm{O}$ \\
\hline
\end{tabular}

